\title{
Activation of a yeast replication origin near a double-stranded DNA break
}

\author{
M.K. Raghuraman, Bonita J. Brewer, and Walton L. Fangman \\ Department of Genetics SK-50, University of Washington, Seattle, Washington 98195 USA
}

Irradiation in the $G_{1}$ phase of the cell cycle delays the onset of DNA synthesis and transiently inhibits the activation of replication origins in mammalian cells. It has been suggested that this inhibition is the result of the loss of torsional tension in the DNA after it has been damaged. Because irradiation causes DNA damage at an undefined number of nonspecific sites in the genome, it is not known how cells respond to limited DNA damage, and how replication origins in the immediate vicinity of a damage site would behave. Using the sequence-specific $\mathrm{HO}$ endonuclease, we have created a defined double-stranded DNA break in a centromeric plasmid in $G_{1}$-arrested cells of the yeast Saccharomyces cerevisiae. We show that replication does initiate at the origin on the cut plasmid, and that the plasmid replicates early in the $S$ phase after linearization in vivo. These observations suggest that relaxation of a supercoiled DNA domain in yeast need not inactivate replication origins within that domain. Furthermore, these observations rule out the possibility that the late replication context associated with chromosomal termini is a consequence of DNA ends.

[Key Words: initiation; replication origin; DNA damage; timing; Saccharomyces cerevisiae; telomere; torsional tension]

Received September 3, 1993; revised version accepted January 13, 1994.

Ionizing radiation given in the $G_{1}$ or $S$ phase of the cell cycle inhibits DNA synthesis (Painter and Young 1987; Siede et al. 1993). Low doses of radiation cause a decrease in the incorporation of radioactive nucleotide precursors into small DNA fragments but not larger fragments, indicating that it is mainly the initiation of DNA replication that is inhibited (Watanabe 1974). Furthermore, when a portion of the chromosomal DNA in a cell is targeted for breakage using bromodeoxyuridine (BrdU) substitution and irradiation with $313 \mathrm{~nm}$ light, replication initiation is blocked in BrdU-substituted regions of the genome but proceeds normally in unsubstituted (and hence undamaged) regions (Povirk 1977). This result suggests that initiation is suppressed specifically in those regions of DNA that are damaged.

The target size for inhibition of the initiation of DNA synthesis is 300-1500 kb (Painter and Young 1975, 1976; Povirk and Painter 1976; Povirk 1977). Because replication origins are spaced $\sim 100 \mathrm{~kb}$ apart (Huberman and Riggs 1968; Hand 1978), it appears likely that a single DNA break can inactivate a cluster of coordinately regulated replication origins (Huberman and Riggs 1968; Painter and Young 1975, 1976; Hand 1978). To account for the effect of a DNA break over large chromosomal distances, it has been suggested that clusters of replicons might reside in a single topological domain. Thus, the loss of superhelical tension after DNA damage could inactivate a cluster of replication origins (Povirk and Painter 1976; Povirk 1977).

A drawback of these studies is that their interpreta- tions rely on statistical analysis of bulk, nascent DNA. It is not known where in the genome the damage has occurred, or even how many sites of damage there are in a given cell; the location of replication origins is not known either. Consequently, it has not been possible to tell whether DNA damage causes inactivation of nearby origins for the duration of the current $\mathrm{S}$ phase, whether origin activation is merely delayed, or whether an origin can become active at its normal time even in the presence of the DNA damage. To address such issues, we made use of a yeast system that delivers DNA damage of defined nature at a defined location. Using an inducible copy of the $H O$ endonuclease from Saccharomyces cerevisiae to cut a single copy plasmid in vivo, we investigated the consequences of a double-stranded DNA break to replication. The $H O$ endonuclease behaves as a restriction enzyme to create a double-strand break at a specific recognition site in the DNA (Kostriken et al. 1983). Hence, after induction of $\mathrm{HO}$ gene transcription, all cells in the population will have a double-stranded DNA break at a defined location. Placing the $H O$ cut site in unique, prokaryotic sequence ensures that the $\mathrm{HO}$ induced breaks created in the $G_{1}$ phase will persist, as DNA breaks in yeast are repaired almost exclusively by recombination with homologous sequences (Brunborg et al. 1980).

We could envision several possible consequences of the DNA break. The presence of the break could cause an arrest at the $G_{1} / S$-phase boundary in a manner analogous to the $R A D 9$-dependent $\mathrm{G}_{2}$ arrest. In addition, the 
cells could modulate initiation of replication from the replication origin on the plasmid in response to the break. The presence of replication intermediates can be detected by the two-dimensional agarose gel method (Brewer and Fangman 1987). We reasoned that failure to detect replication intermediates arising on plasmid cut in vivo would indicate inactivation of the plasmid origin by the DNA break. In contrast, the presence of replication intermediates on the cut plasmid would mean that a replication origin in cis to a break can still be activated, and hence that torsional tension at an origin may not be necessary for its activation.

The presence of replication intermediates on the cut plasmid would not rule out the possibility of delayed activation of the plasmid origin. We know from previous work that chromosomal termini in yeast provide a context that leads to activation in late $\mathrm{S}$ phase of replication origins located nearby (Ferguson et al. 1991; Ferguson and Fangman 1992; Brewer and Fangman 1993). This late activation requires a chromosome end, as authentic telomeres cause a 6- to 10-fold greater delay in origin activation than does an internal tract of telomeric $\left(\mathrm{C}_{1-3} \mathrm{~A}\right)_{\mathrm{n}}$ sequence (Ferguson and Fangman 1992). Does this context effect require a specific telomeric sequence and the resulting specialized telomeric structure, or is it the consequence of some property of a DNA end? Because the in vivo cut plasmid acquires ends but not telomeres, we can distinguish between these two models by determining the time of replication of the cut plasmid. If the cut plasmid replicates early in S phase, then telomeric sequences must be required for the late replication context; if it replicates late, then DNA ends alone must be sufficient to create the late replication context. Delayed activation of a replication origin near a DNA break would be of additional interest because in higher eukaryotes early replication may be required for transcriptional activation of tissue-specific genes (Gottesfeld and Bloomer 1982; Goldman et al. 1984; Riggs and Pfeifer 1992), and damage-dependent late activation of replication origins could, therefore, alter the program of expression of developmentally regulated genes.

Here we show, first, that the presence of doublestranded DNA breaks does not prevent cells from entering S phase; second, that the replication origin on the plasmid remains active after the plasmid has been cut; and third, that the cut plasmid is replicated early in S phase, indicating that DNA ends alone cannot create a late activation context in the absence of telomeric sequences.

\section{Results}

\section{Plasmid linearization in vivo}

The feasibility of this study required that the plasmid be cut to completion by the $\mathrm{HO}$ endonuclease, and that the cut plasmid molecule not be degraded immediately. Plasmid YCpHOCUT4 (Fig. 1A) contains the HO gene under control of the GAL1O transcription promoter, as well as the cut site for the $\mathrm{HO}$ endonuclease. In addition,

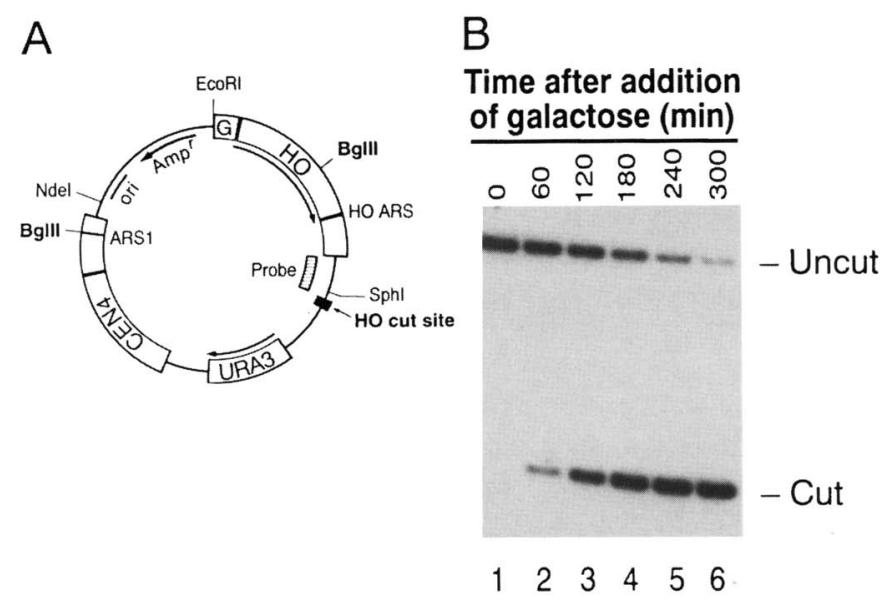

Figure 1. Linearization of plasmid in vivo. (A) Schematic representation of plasmid YCpHOCUT4. Prokaryotic vector sequences are represented by a thin line. The GAL10 promoter (G) and the core consensus sequences of $A R S 1$ and the HO ARS are indicated. $(B)$ Autoradiograph of a Southern blot analysis showing in vivo cutting of YCpHOCUT4 in cells of strain AR120. Cells grown at $23^{\circ} \mathrm{C}$ in minimal medium containing glycerol were arrested in the $G_{1}$ phase by incubation in the presence of $0.2 \mu \mathrm{M} \alpha$-factor for one population doubling time $(=7.75 \mathrm{hr})$. DNA was extracted from control, untreated cells (lane 1) or from cells that were harvested at hourly intervals after addition of galactose (final concentration $=2 \%$ ) to the culture (lanes 2-6). BgIII-digested DNA was blotted to a nylon membrane after electrophoresis through a $1 \%$ agarose gel. The DNA was hybridized with a probe unique to prokaryotic vector sequence (hatched arc in $A$ ). The positions in the gel of the $6.9-\mathrm{kb}$ uncut DNA fragment and the 2-kb HO-cut fragment are indicated.

it has the URA3 gene and two potential origins of replication-ARS1 and the HO ARS. We included a centromere on the plasmid to maintain a low copy number, thereby reducing the likelihood of an uncut plasmid serving as a template for repair of a cut plasmid. Yeast cells transformed with YCpHOCUT4 were grown in minimal medium containing glycerol and arrested in the $\mathrm{G}_{1}$ phase of the cell cycle with $\alpha$-factor. $H O$ gene expression was induced by the addition of galactose. To assess plasmid linearization, DNA prepared from samples taken at various times was cut with a restriction enzyme, transferred to a membrane after gel electrophoresis, and hybridized to a probe flanking the $H O$ cut site. Typically, the plasmid was cut to $\geqslant 95 \%$ within $5 \mathrm{hr}$ after the addition of galactose, and cut plasmid appeared to be stable for several hours at the $\alpha$-factor arrest (Fig. 1B). The $H O$ endonuclease also cuts the DNA at the MAT locus. This break is repaired within an hour or so of the initial cut by the normal mechanism of mating type switching (Connolly et al. 1988). The galactose-induced expression of the $H O$ gene in the experiments described here occurs late in the $G_{1}$ phase, around the same time in the cell cycle that the MAT locus is normally cut and repaired during mating type switching in homothallic strains of yeast (Jensen and Herskowitz 1984). 


\section{S-phase kinetics after plasmid cutting}

Cells respond to ionizing radiation by delaying the onset of $S$ phase or by arresting in the $G_{2}$ phase of the cell cycle until the DNA is repaired (Lavin and Schroeder 1988; Hartwell and Weinert 1989). Does the single HO-induced break cause a delay in entry into or completion of $S$ phase? We compared the cell cycle kinetics of galactose-treated cells with those of control, untreated cells. A culture of cells was arrested with $\alpha$-factor and galactose was added to one-half. In this and subsequent experiments, additional $\alpha$-factor was added at intervals to maintain the arrest for prolonged periods, and glucose was added to cultures at the end of the period of induction with galactose. The glucose serves both to repress $H O$ gene transcription (thereby allowing repair of the $M A T$ locus) and to eliminate physiological differences between the control and the galactose-treated cultures. Because cells quickly adapt upon shifting to richer growth medium (Rivin and Fangman 1980), the addition of glucose also has the effect of overcoming any potential effect of galactose on replication. To release the cells from the arrest rapidly and synchronously, Pronase was added to the cultures to degrade the $\alpha$-factor.

Cells containing $\mathrm{HO}$-cut DNA progressed through $\mathrm{S}$ and entered the $G_{2}$ phase with kinetics identical to those of control cells that had not had the HO gene induced (Fig. 2). We conclude that cells enter and complete $S$ phase with the normal kinetics despite the presence of HO-induced DNA breaks. It is possible that in the ab-

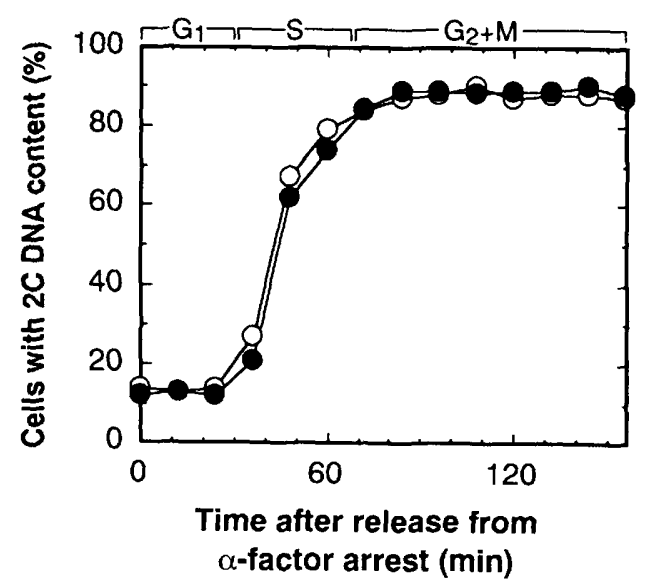

Figure 2. S-phase kinetics of cells containing cut DNA. Cells grown in minimal medium with glycerol as the carbon source were arrested with $\alpha$-factor (see Fig. 1 legend). The culture was split in two and galactose was added to one portion. Additional $\alpha$-factor was added to maintain the arrest (see text), and samples were taken at hourly intervals for Southern blot analysis (not shown/ to determine the extent of plasmid linearization. After 6 $\mathrm{hr}$, glucose $(2 \%)$ was added to both portions (see text) and incubation was continued for another hour. Pronase was then added to both cultures and samples were withdrawn at 12-min intervals. The samples were stained and the DNA content was measured by flow cytometry. The relative proportions of cells with $a_{2}$ DNA content containing uncut $(O)$ and cut $(0 /$ plasmid are plotted. The extent of plasmid linearization was $\geqslant 95 \%$ in this experiment (data not shown). sence of a synchrony regime, DNA breaks cause a transient delay of entry into $S$ phase, as has been observed in mammalian cells that have undergone DNA damage (Povirk and Painter 1976), but that in this experiment, the cells adapt to the DNA damage during incubation in $\alpha$-factor. One way to reveal a $G_{1} \rightarrow S$ phase delay might be to induce $H O$ gene expression in $\mathrm{G}_{2}$-arrested cells, and monitor the kinetics of $G_{1} \rightarrow S$ phase progress in the subsequent cell cycle. However, to overcome the $G_{2}$ arrest induced by the DNA break, the experiment would have to be done in a rad9- strain. In the absence of the $R A D 9$ checkpoint, the results on entry into $S$ phase may not be meaningful.

\section{Protection of ends during $\alpha$-factor arrest}

We noted a slight delay in progress of cells containing cut DNA into mitosis (data not shown). The transient nature of the $G_{2}$ arrest suggested that although the cut plasmid appeared to be protected from degradation in cells arrested with $\alpha$-factor, it was degraded by the time the cells reached the $M$ phase. Complete degradation of the cut plasmid in a cell would presumably eliminate the signal that activates the $R A D 9$ checkpoint and thereby release the cells from the $G_{2}$ arrest. Therefore, we examined the stability of cut plasmid at later stages of the cell cycle by inducing $\mathrm{HO}$ gene transcription in a culture of $\alpha$-factor-arrested cells, adding Pronase to a portion of the culture to release it from the block, and collecting cell samples at various times thereafter. To measure the relative amount of plasmid remaining in each sample, the amount of radioactive probe hybridized to plasmid sequence (on a Southern blot) was compared with the hybridization to chromosomal $A R S 1$-adjacent sequence. Neither plasmid nor chromosomal sequences are replicated in $\alpha$-factor-arrested cells ("-Pronase" in Fig. 3), whereas both plasmid and chromosomal sequences are replicated (see later) in cells released from the block ("+ Pronase"). A change in the relative amounts of hybridization to plasmid and chromosomal sequences can, therefore, be ascribed to changes in the relative abundance of the two sequences in the samples.

When cells left the $G_{1}$ phase (Fig. 3A), cut plasmid was degraded extensively and at a high rate after an initial lag (Fig. 3B,C). In an asynchronously growing population of cells, ends created by $\mathrm{HO}$ endonuclease are usually degraded by a $5^{\prime}$ exonuclease at an average rate of $60-120$ nucleotides/min (White and Haber 1990; Fishman-Lobell et al. 1992). We estimate from the rate of disappearance of full-length molecules that a similar degradation rate is eventually achieved during the $S$ phase in synchronously growing cells. In contrast, the cut plasmid remains largely intact for more than $2 \mathrm{hr}$ in $\alpha$-factorarrested cells as judged by the intensity and sharpness of the band in the gel (Fig. 3C, lanes 12-21). In addition, the $B g I I I$ restriction enzyme site $\sim 2600$ bp from the $H O$ cut site (see Fig. 1) is not converted rapidly to single-stranded DNA by exonuclease, as the site remains susceptible to cleavage by $B g I I I$ for $\geqslant 3 \mathrm{hr}$ (data not shown). On the basis of these observations, we estimate that the rate of exonuclease action in cells arrested with $\alpha$-factor is $\leqslant 10$ 
A
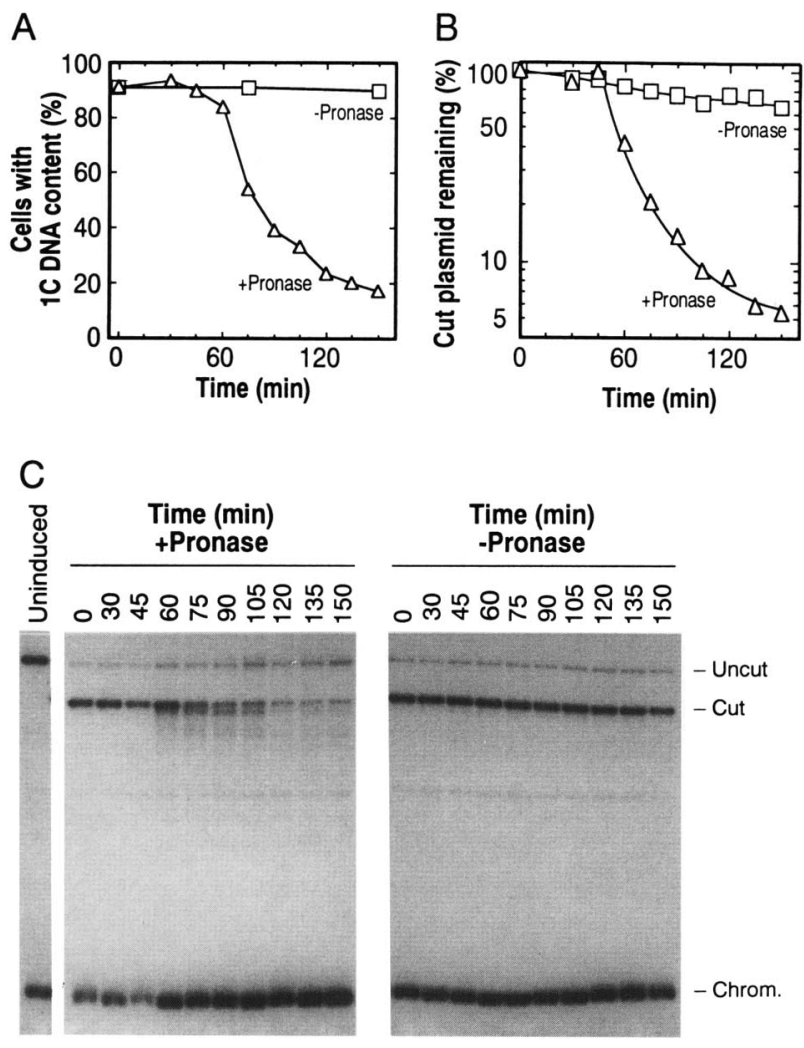

$\begin{array}{lllllllllll}1 & 2 & 3 & 4 & 5 & 6 & 7 & 8 & 9 & 10 & 11\end{array}$

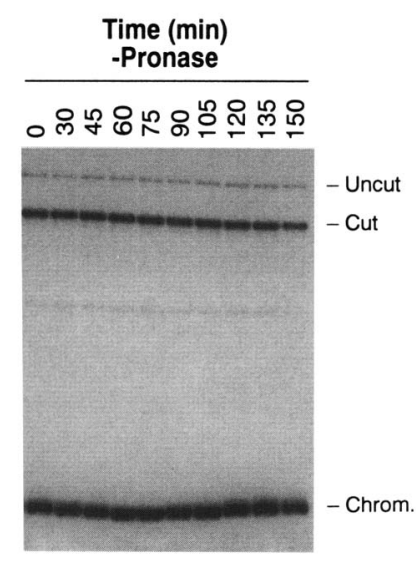

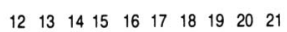

nucleotides/min. A greater stability of $H O$-generated ends has also been reported for cells arrested in $\mathrm{S}$ phase with hydroxyurea (Connolly et al. 1988). We note that there is a parallel between plasmid degradation and the process of double-strand break repair: the rate of gap repair is also greatly reduced in $G_{1}$-arrested cells (FishmanLobell et al. 1992). These observations suggest that additional processing enzymes are activated during $S$ phase. Alternatively, the accessibility of chromatin to nucleases may increase as the cells enter $S$ phase. Because cut plasmid is also protected from degradation in $\mathrm{G}_{2}$-arrested cells (data not shown), resistance of broken DNA to degradation is not a property peculiar to cells treated with $\alpha$-factor.

Recently it has been reported that HO-mediated cutting of a dispensable plasmid in vivo causes $40-50 \%$ of the cells in the population to arrest irreversibly in the $G_{1}$ or $G_{2}+M$ phases (Bennet et al. 1993). However, we find that the viability of cells is undiminished after induction of $\mathrm{HO}$ gene transcription for $6 \mathrm{hr}$ in the $\mathrm{G}_{1}$ phasewhereas $77 \%$ of the cells in the culture failed to form colonies on medium lacking uracil (presumably because the plasmid was degraded), as many cells formed colonies on medium supplemented with uracil as did control, uninduced cells. This difference may stem from differences between the strains used, or be the result of incubation in $\alpha$-factor.

\section{Origin activity on a plasmid cut in vivo}

If an origin located in cis to a DNA break can initiate

Figure 3. Cell cycle-dependent degradation of plasmid linearized in vivo. Cells of strain AR120 [YCpHOCUT4] grown in glycerol as the carbon source were arrested with $\alpha$-factor /see Fig. 1 legend), and galactose was added to the medium. Five hours later, glucose was added to repress $H O$ gene expression, and the culture was split in two. One portion received an additional dose of $\alpha$-factor, whereas the other was treated with Pronase (final concentration $=0.1 \mathrm{mg} / \mathrm{ml}$ ) to release the culture from the $\alpha$-factor block. Samples were taken for flow cytometry $|A|$ and for Southern hybridization analysis of the DNA $(B, C)$ before addition of galactose, at the time of Pronase addition, and at various times thereafter. $(A)$ Cell cycle progress of cells released from $\alpha$-factor arrest $(\Delta)$ by addition of Pronase (at time $=0 \mathrm{~min}$ ). Control cells $(\square)$ that were not Pronase treated remained in $G_{1}$ through the course of the experiment. Samples were analyzed by flow cytometry; the proportion of cells in the $G_{1}$ peak of each sample is plotted. $(B)$ Plot showing the ratio of cut, full-length plasmid to an $A R S 1$-adjacent chromosomal DNA fragment in cells arrested in $\mathrm{G}_{1}(\square)$ and cells released from the arrest $(\Delta)$ normalized to the ratio observed at the time of Pronase addition (see text). The radioactivity in bands corresponding to the $7.5-\mathrm{kb}$ cut plasmid fragment and the $1.28-\mathrm{kb}$ $A R S 1$-adjacent chromosomal DNA fragment in blot $(C)$ were quantitated by PhosphorImaging. $(C)$ Autoradiograph of Southern blots yielding the data in $B$. DNA extracted from the samples was cut with EcoRI, fractionated by electrophoresis, and transferred to a membrane. The blot was hybridized simultaneously to two probes: an NdeI-EcoRI fragment that includes the $\beta$-lactamase gene (see Fig. 1A) and is thus specific for the plasmid, and a fragment specific to sequences adjacent to ARS1 on chromosome IV. (Lane 1) DNA obtained from cells before addition of galactose; (lanes 2-11) DNA from cells collected at the time of Pronase addition (lane 2), or at 15-min intervals starting $30 \mathrm{~min}$ after the addition of Pronase (lanes 3-11); (lanes 12-21) the corresponding samples from the control culture incubated in $\alpha$-factor. The positions in the gel of uncut and cut plasmid and of the ARS1-adjacent chromosomal fragment ("Chrom.") are indicated.

replication, then in vivo cut plasmid obtained from $S$ phase cells should contain replication bubbles. Accordingly, we looked for bubble forms of replication intermediates on the cut plasmid using two-dimensional gel electrophoresis (Brewer and Fangman 1987). To simplify interpretation of the results, we used a plasmid that has only one potential replication origin, the $H O$ ARS (Fig. 4A).

DNA was isolated from untreated control cells and from cells in which $H O$ gene expression had been induced. In this experiment there was some uncut plasmid after galactose induction of the $H O$ gene, providing an internal control for origin activity on the cut plasmid. After digestion with $\mathrm{BgIII}$ and $\mathrm{NcOI}$ and two-dimensional agarose gel electrophoresis (Brewer and Fangman 1987), the DNA was transferred to a membrane and hybridized to a fragment of the plasmid immediately adjacent to the HO cut site (Fig. 4A). Because the DNA fragment being probed contains an asymmetrically placed origin of replication, a bubble-to-Y hybridization pattern (Brewer and Fangman 1987) was seen for the control, uncut plasmid (Fig. 4B). In DNA prepared from cells in which some of the plasmid had been cut in vivo a second bubble-to-Y 


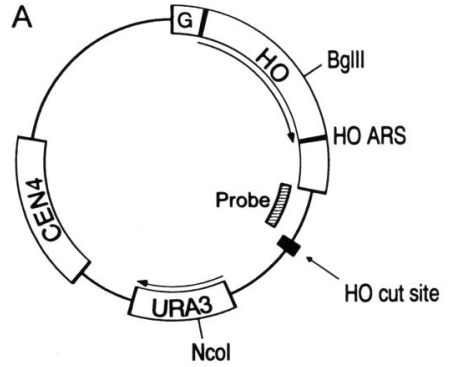

Figure 4. Origin activity on cut and uncut plasmids. Strain AR120 [YCpHOCUT4DARS1] was grown in glycerol and arrested with $\alpha$-factor (see Fig. 1 legend), and the culture was split in two. Galactose was added to one portion; after $5.5 \mathrm{hr}$, glucose was added to both cultures. The cells were synchronized further by arrest at the $c d c 7$ block after release from the $\alpha$-factor arrest, and DNA was extracted from pooled S-phase culture samples after release from the $c d c 7$ block (Huberman et al. 1987; Brewer et al. 1992). (A) Schematic representation of plasmid YCpHOCUT4 $\triangle A R S 1$. This plasmid differs from YCpHOCUT4 (Fig. 1A) in that $A R S 1$ has been deleted. $(B, C)$ Two-dimensional agarose gels of uncut and cut plasmid. The cartoons (left in $B, C$ ) depict the bubble-to- $Y$ two-dimensional gel patterns expected for an origin that is asymmetrically placed in the DNA fragment of uncut and cut plasmids, respectively (Brewer and Fangman 1987). DNA (3-7 $\mu \mathrm{g}$ ) from control (Uncut) and galactosetreated cells (Cut) was digested with BgIII and Ncol. After two-dimensional agarose gel electrophoresis and transfer to a membrane, the DNA was hybridized to a probe derived from prokaryotic vector sequence (hatched $\operatorname{arc}$ in $A$ ).
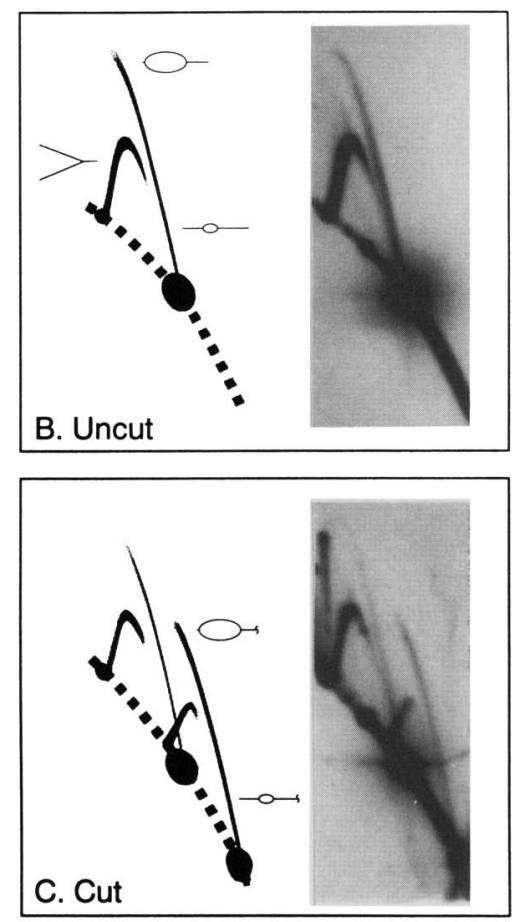

pattern appeared, arising from the lower monomer spot of HO-cut linear molecules (Fig. 4B). Note that in the in vivo cut molecule, the bubble-to-Y transition occurs only when the DNA fragment is almost fully replicated, as expected because the HO ARS becomes more symmetrically placed in the restriction fragment being probed. We conclude from these observations that the HO ARS can become active even when located in cis to a double-stranded DNA break. Moreover, no significant amount of aberrant replication is initiated at the DNA breaks on the molecule. Such events would have been signaled by a complete simple $\mathrm{Y}$ arc.

\section{Replication time of cut plasmid}

Because an origin located near a DNA break is activated in S phase, we could use our test plasmid to distinguish between two alternative models for the late activation of origins located close to chromosomal termini. It was clear from previous work that a chromosomal end creates a context that causes late activation of replication origins (Ferguson et al. 1991; Ferguson and Fangman 1992). However, it was not clear whether a specific $\left(\mathrm{C}_{1-3} \mathrm{~A}\right)_{\mathrm{n}}$ telomeric sequence-dependent structure was required, or whether some property of a free DNA end was responsible for the late replication context. If the latter possibility were true, then the linearized plasmid should replicate late in S phase, around the same time as a telomeric, linear form of the plasmid. If, on the other hand, the late replication context is created only when a sequence-dependent telomeric structure is present at the ends, then the linearized plasmid (lacking any telomeric sequence) should replicate early in S phase, around the same time as uncut, circular plasmid.

Two controls were necessary to establish the baseline of early- and late-replicating forms of the test plasmid. First, we needed to determine the replication kinetics of uncut YCpHOCUT4. On the basis of previous work /Ferguson and Fangman 1992), we expected that the uncut plasmid would replicate early in the S phase. Second, we needed to show that when YCpHOCUT4 is converted to a genuine linear minichromosome with telomeres, its origins are subject to the late activation context. The linear minichromosome YCpHOCUT4-LIN was therefore created by insertion of telomeric $\left(\mathrm{C}_{1-3} \mathrm{~A}\right)_{n}$ sequences at the SphI site of YCpHOCUT4 (see Materials and methods).

The kinetics of plasmid replication were determined by density transfer experiments (McCarroll and Fangman 1988; Ferguson et al. 1991). For the two controls, cultures of strains transformed with YCpHOCUT4 or YCpHOCUT4-LIN were synchronized by sequential arrests, first in $G_{1}$ with $\alpha$-factor, then at the $G_{1} / S$ boundary by incubation at $37^{\circ} \mathrm{C}$, the restrictive temperature for the $c d c 7^{\text {ts }}$ mutation. Sequences adjacent to ARS1 on chromosome IV were used as a marker for early replication (McCarroll and Fangman 1988), and restriction fragment R11, centromere-proximal to ARS501 on chromosome V (see Ferguson et al. 1991), as a marker for late replication. To examine the kinetics of replication of in vivo cut YCpHOCUT4, the culture was synchronized with $\alpha$-factor alone as cut plasmid undergoes some degradation between the $\alpha$-factor arrest and the $c d c 7$ arrest. The time scale for replication when $\alpha$-factor alone is used for synchronization (Fig. 5C) is different from that of the control experiments (Fig. 5A,B). However, in a separate cutting experiment that included both synchrony steps, where the proportion of cut plasmid had dropped to $60-70 \%$ by the time the cells entered $S$ phase, the relative replica- 
A Uncut circular plasmid

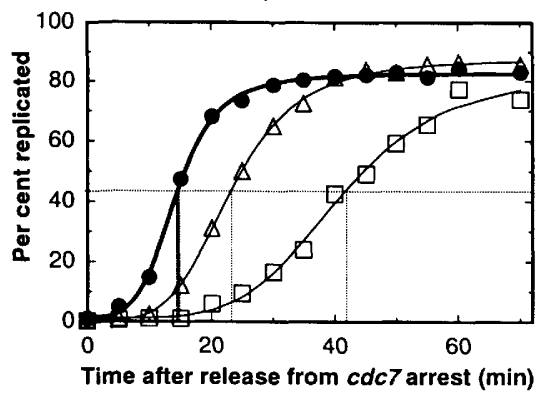

B Telomeric linear plasmid

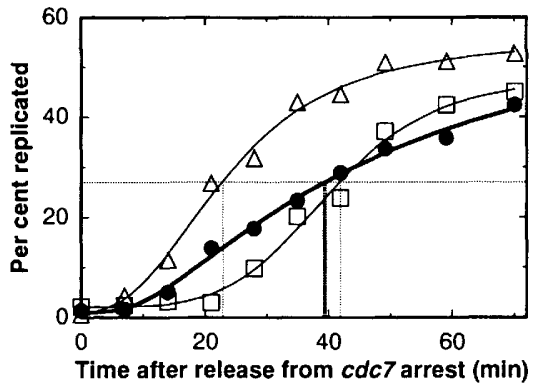

C Cut plasmid

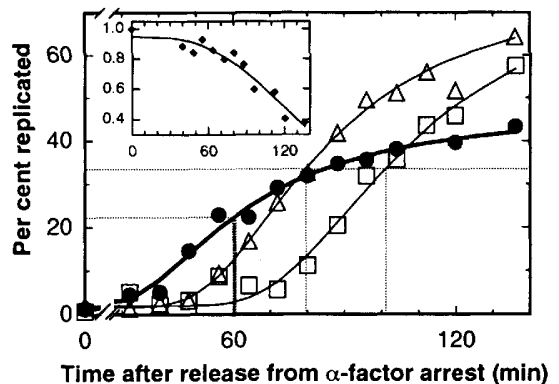

Figure 5. Replication kinetics of circular and linear plasmids. The replication kinetics of plasmid (O), early-replicating chromosomal ARS1 sequence $(\Delta ;$ McCarroll and Fangman 1988) and late-replicating chromosome V fragment $(\square ; \mathrm{R} 11$ fragment, Ferguson et al. 1991) are shown. The broken line shows the approximate time of half-maximal replication of each sequence. $(A)$ Replication of uncut YCpHOCUT4; $(B)$ replication of YCpHOCUT4-LIN, a linear version of YCpHOCUT4 containing telomeres at the SphI site (see Materials and methods); $(C)$ replication of YCpHOCUT4 linearized in vivo. Cutting of the plasmid was $\geqslant 95 \%$ complete in this experiment (data not shown). (Inset) The kinetics of plasmid degradation ( $\mid$ ) are shown by the ratio of plasmid to chromosomal $A R S 1$-adjacent sequence, normalized to the first sample.

tion kinetics of plasmid and markers (data not shown) were comparable with those shown in Figure 5C. Likewise, when the control strain with uncut YCpHOCUT4 was used in an experiment with $\alpha$-factor as the sole synchronizing step, the relative replication kinetics were comparable with those seen when both $\alpha$-factor and $c d c 7$ were used (Fig. 5A; data not shown). In general, we find that the $t_{\text {rep }}$ (time of half-maximal replication, adjusting for the population of cells that fails to enter S phase) of a given sequence may vary with the experiment, but the differences in $t_{\text {rep }}$ values between sequences are reproducible.

Relative to the early- and late-replicating markers, uncut circular YCpHOCUT4 replicated before chromosomal $A R S 1$, very early in the $\mathrm{S}$ phase (Fig. 5A), and the telomeric linear plasmid YCpHOCUT4-LIN replicated much later, at about the same time as R1l (Fig. 5B). Therefore, both control plasmids behave as expected from previous work (Ferguson and Fangman 1992), and serve as guideposts for comparison with the cut plasmid.

In vivo-cut YCpHOCUT4 replicated early in S phase, before chromosomal ARS1 (Fig. 5C). Although a precise determination of $t_{\text {rep }}$ for the cut plasmid is complicated by the apparent low extent of replication of the cut plasmid, it is clear that as with the intact, circular plasmid, $50 \%$ of its replication was completed before $50 \%$ of the replication of chromosomal ARS1 sequence was completed (Fig. 5A,C). These kinetics contrast with the replication kinetics of the telomeric linear plasmid (Fig. 5B), which completed $50 \%$ of its replication well after chromosomal ARS1 sequence, around the same time as the R11 late replication marker. We interpret the apparently low extent of replication of cut plasmid to be a consequence of its degradation. Substantial $(\sim 25-30 \%)$ replication of the plasmid occurs before the onset of degradation /as judged by the amount of hybridization to plasmid sequences compared with chromosomal sequences; Fig. 5C inset). Therefore, we believe that replication of the cut plasmid precedes its degradation. Thus, the plas- mid in a cell would likely be replicated before the onset of degradation, and the pool of hybrid density plasmid molecules would be depleted preferentially, depressing the apparent extent of replication of the cut plasmid. When adjusted for degradation of the hybrid density plasmid molecules, the extent of replication of cut plasmid becomes comparable with that of the marker sequences (not shown). Note that although the kinetics of plasmid degradation closely follow those of plasmid replication, we do not believe that replication of the plasmid is necessary for its rapid degradation, because a nonreplicating fragment of DNA is also degraded in S phase with similar kinetics (K. Kolor, M.K. Raghuraman, B. Brewer, and W.L. Fangman, unpubl.).

The early replication of the in vivo linearized plasmid is consistent with a model that a specific telomeric structure, requiring specific telomeric sequences present at chromosomal ends, is responsible for the late replication context, and rules out the model that some sequence-independent property associated with DNA ends causes the late replication context seen in the telomeric linear form of the plasmid.

\section{Discussion}

\section{Origin activation in the presence of a DNA break}

The results described here show that replication origins can be activated in a cell that contains a unique doublestranded DNA break. Because cells containing cut plasmid enter and complete $\mathrm{S}$ phase with normal kinetics (Fig. 2), the general program of chromosomal replication-and hence, origin activation-must continue even in the presence of the DNA damage. This observation contrasts with previous observations that DNA synthesis is apparently delayed upon irradiation of $G_{1}$ cells (Painter and Young 1987; Siede et al. 1993). The difference in response to radiation versus an $\mathrm{HO}$-induced break may stem from the differing extents of DNA dam- 
age. Indeed, there is some correlation between the radiation dose and the magnitude of the response (Leeper et al. 1973; Watanabe 1974), suggesting that very low levels of damage may fall below the threshold of detection by a cell in $\mathrm{G}_{1}$. Alternatively, yeast cells containing broken DNA may adapt to the damage during the synchronization procedure. This possibility would be difficult to test experimentally: Because in vivo cutting takes a finite amount of time, it would have to precede or accompany the synchronization step, creating the opportunity for the cells to adapt to the broken DNA either during the synchronizing block or during recovery from the block.

A perhaps more striking result is that an origin located in cis to a DNA break (and, in this instance, only $\sim 1 \mathrm{~kb}$ from the break) can be activated in $S$ phase. Because the HO-mediated break presumably eliminates superhelical tension on the plasmid, the implication of this result is that torsional tension is not required for origin activation. The observation that the presence of a torsional sink in the form of an easily unwound sequence does not prevent ARS function on a plasmid (Umek and Kowalski 1990) indirectly supports this interpretation.

How, then, does one explain the apparent inactivation of replication origins after irradiation of mammalian cells (Watanabe 1974; Painter and Young 1975, 1976; Povirk and Painter 1976; Povirk 1977)? Could the cut plasmid, being an extrachromosomal element, have different topological requirements for origin activation than authentic chromosomes? The observation that a broken test chromosome in yeast can be propagated for several generations (Sandell and Zakian 1993) argues against this idea. Could the $H O$ plasmid somehow behave as a covalently closed circle even after it has been cut? This possibility also seems unlikely, both because the $\mathrm{HO}$ endonuclease does not covalently bind the DNA at the site of cleavage (Raveh et al. 1989), and because the $5^{\prime}$ ends created by the $H O$ endonuclease are susceptible in vivo to attack by exonuclease (Fishman-Lobell and Haber 1992; Fishman-Lobell et al. 1992; Sugawara and Haber 1992). Consequently, even if the $3^{\prime}$ ends were held together by the endonuclease, the $5^{\prime}$ ends would be free to swivel. Sandell and Zakian (1993) have proposed cycles of end fusion and chromosome breakage as a possible mechanism for the unstable propagation of a broken test chromosome. Because we see no evidence by Southern blotting of end fusion after plasmid cutting, we can rule out the possibility that fused, covalently closed forms of the plasmid are created. We therefore believe that $H O$ cleavage relaxes the plasmid, and hence, that superhelical tension is not required for origin function. A more plausible explanation for the initiation block in $\mathrm{X}$-ray-irradiated mammalian cells is that the block is not directly caused by a loss of torsional tension; rather, the radiation-induced breaks signal a control mechanism that then suppresses origin activity in the vicinity of the break (Hartwell and Weinert 1989). The radio-resistant initiation of DNA synthesis seen in the genetic disease ataxia-telangiectasia is believed to be a consequence of a defect in such a control mechanism (Painter and Young 1980|. As with the cell cycle delay, there might be a threshold level of damage below which no response is elicited.

\section{Time of origin activation on cut plasmid}

Previous work showing that chromosomal termini are 6to 10 -fold more effective at creating a late replication context than internal $\left|C_{1-3} A\right\rangle_{n}$ sequences had suggested two alternative mechanisms for the context effect-a sequence-dependent telomeric structure could be responsible, or a sequence-independent property of a DNA end could be responsible. Our observation that the cut plasmid replicates early in S phase (Fig. 5, cf. B and C) rules out the latter model. By inference, therefore, the late replication context associated with chromosomal termini must be the consequence of some sequence-specific telomeric structure. It seems likely that the specialized chromatin structure at telomeres (Wright et al. 1992) plays a role in creating this context. Chromatin structure has been implicated in the silencing of genes close to telomeres in yeast (Gottschling 1992), and proteins important for silencing colocalize with the telomere-binding protein RAP1 (Palladino et al. 1993). Although the transcriptional silencing effect does not spread as far from the telomere as does the late replication context (Aparicio et al. 1991; Ferguson et al. 1991; Renauld et al. 1993|, it is attractive to think that there may be a common underlying mechanism.

\section{Materials and methods}

\section{Plasmids}

Plasmid YCpHOCUT4 was constructed by insertion of the 123-bp HO cut site (Kostriken et al. 1983) at the SalI site of pGAL-HO (Jensen and Herskowitz 1984). Plasmid YCpHOCUT4 4 ARS1 was derived from YCpHOCUT4 by deletion of the XhoI-NdeI fragment containing ARS1. To construct YCpHOCUT4-LIN, an SphI fragment of DNA containing two $\sim 280$-bp tracts of $\left(\mathrm{C}_{1-3} \mathrm{~A}\right)_{n}$ telomeric sequence in inverted orientation was obtained from plasmid $\mathrm{pYLPV}_{K}$, a gift from $R$. Wellinger and V. Zakian (Fred Hutchinson Cancer Research Center, Seattle, WA). This DNA fragment was inserted in the SphI site of YCpHOCUT4 (see Fig. 1A). The resulting construct was digested with SacI, which cuts $\sim 10$ bp internal to each telomeric tract, linearizing the plasmid and leaving the telomeric sequences in the proper orientation relative to the ends. Yeast strain AR120 (MATa, ho, cdc7-1, bar1, ura3-52, trp1-289, leu2-3, 112, his6, HMLa, HMRa) was transformed to uracil prototrophy with YCpHOCUT4, YCpHOCUT4 4 ARS1, or YCpHOCUT4-LIN.

\section{Flow cytometry}

Cell samples were mixed with an equal volume of ice-cold 0.2 M EDTA, $0.2 \% \mathrm{NaN}_{3}$, pelleted by centrifugation, stained for flow cytometry as previously described (Hutter and Eipel 1979), and analyzed on a Becton-Dickinson flow cytometer.

\section{Two-dimensional agarose gel electrophoresis}

DNA extracted from pooled S-phase culture samples (Huberman et al. 1987; Brewer et al. 1992) was analyzed by two-di- 
mensional gel electrophoresis as described previously (Brewer and Fangman 1987). The first dimension gel was $0.4 \%$ agarose in $1 \times \mathrm{TBE}$, and the second dimension gel was $1.1 \%$ agarose in $1 \times \mathrm{TBE}, 0.3 \mu \mathrm{g} / \mathrm{ml}$ ethidium bromide.

\section{Determination of replication times}

The density transfer procedure was modified from a previously published method (Ferguson et al. 1991). Cells were grown at $23^{\circ} \mathrm{C}$ for at least seven generations in medium containing $0.1 \%$ $\left[{ }^{13} \mathrm{C}\right]$-sodium acetate and $0.01 \%\left[{ }^{15} \mathrm{~N}\right]$-ammonium sulfate as the sole carbon and nitrogen source, respectively. The cells were arrested with $\alpha$-factor for up to $11 / 4$ population doubling times $(\sim 12 \mathrm{hr})$, filtered and resuspended in isotopically normal minimal medium containing glycerol and $\alpha$-factor and lacking uracil. The culture was shifted to $37^{\circ} \mathrm{C}$ immediately (Fig. $5 \mathrm{~A}$ ) or after an additional $5.5 \mathrm{hr}$ (Fig. $5 \mathrm{~B}$ ) and Pronase (to $0.1 \mathrm{mg} / \mathrm{ml}$ ) was added to allow the cells to progress from the $\alpha$-factor block to the $c d c 7$ block. Alternatively, galactose was added to the culture and incubation was continued for $5.5 \mathrm{hr}$ (Fig. 5C). Cells were released into $\mathrm{S}$ phase by a temperature downshift to $23^{\circ} \mathrm{C}$ (Fig. 5, A and B) or by addition of Pronase to the culture (Fig. 5C). Samples were collected at various times thereafter, and processed for $\mathrm{CsCl}$ density gradient centrifugation as described previously (Ferguson et al. 1991). Hybridization signals were quantitated by PhosphorImaging.

\section{Acknowledgments}

Autoradiographic analysis was carried out by the PhosphorImager Facility of the Markey Molecular Medicine Center at the University of Washington. We are grateful to L. Gilbertson for his gift of plasmid, and R. Wellinger, L. Sandell, and V. Zakian for sharing their telomeric plasmid constructs and results before publication. We thank John Diller, Kathy Friedman, Lee Hartwell, Dan Lockshon, and Terry Ward for their comments on the manuscript. This work was supported by National Institutes of General Medical Sciences grant 18926 to B.J.B. and W.L.F., and by Damon Runyon-Walter Winchell Cancer Research Fund Fellowship DRG-1063 to M.K.R.

The publication costs of this article were defrayed in part by payment of page charges. This article must therefore be hereby marked "advertisement" in accordance with 18 USC section 1734 solely to indicate this fact.

\section{References}

Aparicio, O.M., B.L. Billington, and D.E. Gottschling. 1991. Modifiers of position effect are shared between telomeric and silent mating-type loci in S. cerevisiae. Cell 66: 12791287.

Bennet, C.G., A.L. Lewis, K.K. Baldwin, and M.A. Resnick. 1993. Lethality induced by a single site-specific doublestrand break in a dispensable yeast plasmid. Proc. Natl. Acad. Sci. 90: 5613-5617.

Brewer, B.J. and W.L. Fangman. 1987. The localization of replication origins on ARS plasmids in S. cerevisiae. Cell 51: 463-471.

- 1993. Initiation at closely spaced origins in a yeast chromosome. Science 262: 1728-1731.

Brewer, B.J., D. Lockshon, and W.L. Fangman. 1992. The arrest of replication forks in the rDNA of yeast occurs independently of transcription. Cell 71: 267-276.

Brunborg, G., M.A. Resnick, and D.H. Williamson. 1980. Cellcycle-specific repair of DNA double-strand breaks in Saccha- romyces cerevisiae. Radiat. Res. 82: 547-558.

Connolly, B., C.I. White, and J.E. Haber. 1988. Physical monitoring of mating type switching in Saccharomyces cerevisiae. Mol. Cell. Biol. 8: 2342-2349.

Ferguson, B.M. and W.L. Fangman. 1992. A position effect on the time of replication origin activation in yeast. Cell 68: $333-339$.

Ferguson, B.M., B.J. Brewer, A.E. Reynolds, and W.L. Fangman. 1991. A yeast origin of replication is activated late in $S$ phase. Cell 65: 507-515.

Fishman-Lobell, J. and J.E. Haber. 1992. Removal of nonhomologous DNA ends in double-strand break recombination: The role of the yeast ultraviolet repair gene RAD1. Science 258: $480-484$.

Fishman-Lobell, J., N. Rudin, and J.E. Haber. 1992. Two alternative pathways of double-strand break repair that are kinetically separable and independently modulated. Mol. Cell. Biol. 12: 1292-1303.

Goldman, M.A., G.P. Holmquist, M.C. Gray, L.A. Caston, and A. Nag. 1984. Replication timing of genes and middle repetitive sequences. Science 224: 686-692.

Gottesfeld, J. and L.S. Bloomer. 1982. Assembly of transcriptionally active 5S RNA gene chromatin in vitro. Cell 28: 781-791.

Gottschling, D.E. 1992. Telomere-proximal DNA in Saccharomyces cerevisiae is refractory to methyltransferase activity in vivo. Proc. Natl. Acad. Sci. 89: 4062-4065.

Hand, R. 1978. Eucaryotic DNA: Organization of the genome for replication. Cell 15: 317-325.

Hartwell, L.H. and T.A. Weinert. 1989. Checkpoints: Controls that ensure the order of cell cycle events. Science 246: 629634.

Huberman, J.A. and A.D. Riggs. 1968. On the mechanism of DNA replication in mammalian chromosomes. J. Mol. Biol. 32: 327-341.

Huberman, J.A., L.D. Spotila, K.A. Nawotka, S.M. el-Assouli, and L.R. Davis. 1987. The in vivo replication origin of the yeast $2 \mu \mathrm{m}$ plasmid. Cell 51: 473-481.

Hutter, K.-J. and H.E. Eipel. 1979. Microbial determinations by flow cytometry. J. Gen. Microbiol. 113: 369-375.

Jensen, R.E. and I. Herskowitz. 1984. Directionality and regulation of cassette substitution in yeast. Cold Spring Harb. Symp. Quant. Biol. 49: 97-104.

Kostriken, R., J.N. Strathern, A.J. Klar, J.B. Hicks, and F. Heffron. 1983. A site-specific endonuclease essential for matingtype switching in Saccharomyces cerevisiae. Cell 35: 167174.

Lavin, M.F. and A.L. Schroeder. 1988. Damage-resistant DNA synthesis in eukaryotes. Mutat. Res. 193: 193-206.

Leeper, D.B., M.H. Schneiderman, and W.C. Dewey. 1973. Radiation-induced cycle delay in synchronized Chinese hamster cells: Comparison between DNA synthesis and division. Radiat. Res. 53: 326-337.

McCarroll, R.M. and W.L. Fangman. 1988. Time of replication of yeast centromeres and telomeres. Cell 54: 505-513.

Painter, R.B. and B.R. Young. 1975. X-ray-induced inhibition of DNA synthesis in Chinese hamster ovary, human HeLa, and mouse L cells. Radiat. Res. 64: 648-656.

- 1976. Formation of nascent DNA molecules during inhibition of replicon initiation in mammalian cells. Biochim. Biophys. Acta 418: 146-153.

- 1980. Radiosensitivity in ataxia-telangiectasia: A new explanation. Proc. Natl. Acad. Sci. 77: 7315-7317.

. 1987. DNA synthesis in irradiated mammalian cells. J. Cell. Sci. (suppl.) 6: 207-214.

Palladino, F., T. Laroche, E. Gilson, A. Axelrod, L. Pillus, and 
S.M. Gasser. 1993. SIR3 and SIR4 proteins are required for the positioning and integrity of yeast telomeres. Cell 75: 543-555.

Povirk, L.F. 1977. Localization of inhibition of replicon initiation to damaged regions of DNA. J. Mol. Biol. 114: 141-151.

Povirk, L.F. and R.B. Painter. 1976. The effect of 313 nanometer light on initiation of replicons in mammalian cell DNA containing bromodeoxyuridine. Biochim. Biophys. Acta 432: 267-272.

Raveh, D., S.H. Hughes, B.K. Shafer, and J.N. Strathern. 1989. Analysis of the HO-cleaved MAT DNA intermediate generated during the mating type switch in the yeast Saccharomyces cerevisiae. Mol. Gen. Genet. 220: 33-42.

Renauld, H., O.M. Aparicio, P.D. Zierath, B.L. Billington, S.K. Chhablani, and D.E. Gottschling. 1993. Silent domains are assembled continuously from the telomere and are defined by promoter distance and strength, and by SIR3 dosage. Genes \& Dev. 7: 1133-1145.

Riggs, A.D. and G.P. Pfeifer. 1992. X-chromosome inactivation and cell memory. Trends Genet. 8: 169-174.

Rivin, C.J. and W.L. Fangman. 1980. Replication fork rate and origin activation during the $\mathrm{S}$ phase of Saccharomyces cerevisiae. J. Cell Biol. 85: 108-115.

Sandell, L.L. and V.A. Zakian. 1993. Loss of a yeast telomere: Arrest, recovery, and chromosome loss. Cell 75: 729-739.

Siede, W., A.S. Friedberg, and E.C. Friedberg. 1993. RAD9-dependent Gl arrest defines a second checkpoint for damaged DNA in the cell cycle of Saccharomyces cerevisiae. Proc. Natl. Acad. Sci. 90: 7985-7989.

Sugawara, N. and J.E. Haber, 1992. Characterization of doublestrand break-induced recombination: Homology requirements and single-stranded DNA formation. Mol. Cell. Biol. 12: $563-575$.

Umek, R.M. and D. Kowalski. 1990. The DNA unwinding element in a yeast replication origin functions independently of easily unwound sequences present elsewhere on a plasmid. Nucleic Acids Res. 18: 6601-6605.

Watanabe, I. 1974. Radiation effects on DNA chain growth in mammalian cells. Radiat. Res. 58: 541-556.

White, C.I. and J.E. Haber. 1990. Intermediates of recombination during mating type switching in Saccharomyces cerevisiae. EMBO /. 9: 663-673.

Wright, J.H., D.E. Gottschling, and V.A. Zakian. 1992. Saccharomyces telomeres assume a non-nucleosomal chromatin structure. Genes \& Dev. 6: 197-210. 


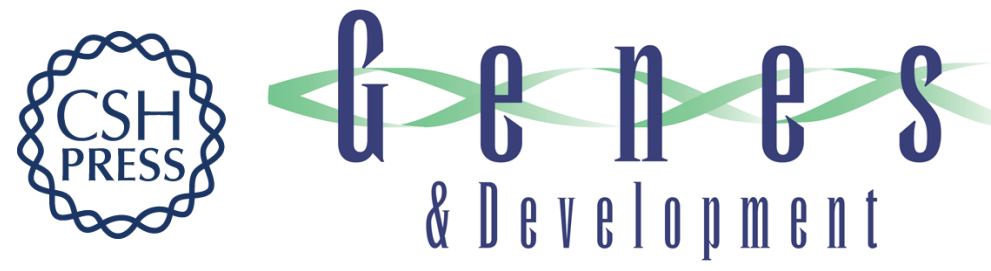

\section{Activation of a yeast replication origin near a double-stranded DNA break.}

M K Raghuraman, B J Brewer and W L Fangman

Genes Dev. 1994, 8:

Access the most recent version at doi:10.1101/gad.8.5.554

References This article cites 42 articles, 15 of which can be accessed free at:

http://genesdev.cshlp.org/content/8/5/554.full.html\#ref-list-1

License

Email Alerting

Service

Receive free email alerts when new articles cite this article - sign up in the box at the top right corner of the article or click here.

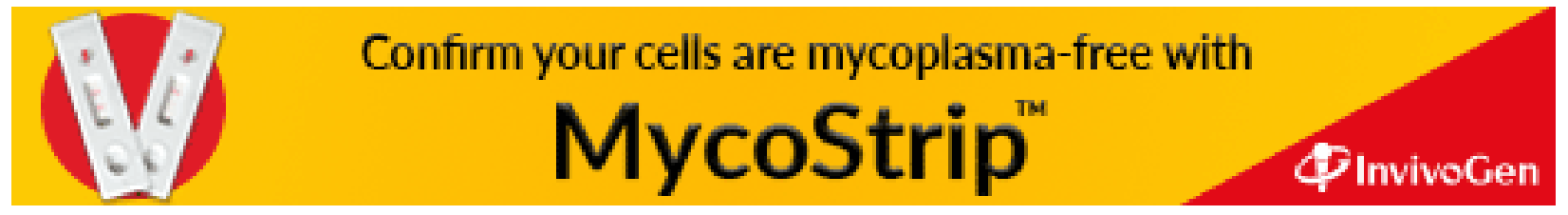

